\title{
Computing the value of flexibility in electricity retail, ahead and balancing markets
}

\author{
Aliene van der Veen \\ Centrum Wiskunde \& Informatica \\ Amsterdam, Science Park 123 \\ Email: veen@cwi.nl
}

\author{
Michael Kaisers \\ Centrum Wiskunde \& Informatica \\ Amsterdam, Science Park 123 \\ Email: kaisers@cwi.nl
}

\begin{abstract}
Renewable generation of energy is becoming more affordable, and is therefore increasingly adopted to match local demand. In addition, storage and demand response solutions have reached the market, which provides flexibility and thereby facilitates intelligent energy management. It has been suggested that this bottom-up flexibility should contribute to the balancing of the future smart grid, but financial incentives have so far been elusive. This article presents computations that assess the profitability of intelligent energy management for three different value propositions: increasing self-sufficiency under typical retail tariffs, or participating in ahead or balancing market price fluctuations, specifically in the Netherlands, Germany and Italy. First, an upper bound valuation of flexibility is computed from 2013 market data. Second, a technology-neutral profit analysis is proposed and demonstrated on selected technologies. Our simulations indicate the highest value for flexibility in the Dutch and German imbalance markets $(7.7 \& 9.4 € \mathrm{c} / \mathrm{kWh})$ and the Dutch reserve market $(10.4 € \mathrm{c} / \mathrm{kWh})$, and for small prosumers in the German and Italian retail markets $(12 \& 10 € \mathrm{c} / \mathrm{kWh})$. Results indicate that there is sufficient value in demand response if activation costs are sufficiently low, which innovative tariffs should exploit to provide incentives for bottom-up balancing of the future smart grid.
\end{abstract}

\section{INTRODUCTION}

With the wide-spread adoption of generation from uncontrollable renewable resources in the electricity system, the need for flexible consumption and production is increasing. Storage and demand response (DR) technologies are considered valuable sources of flexiblility which can be valorized by intelligent energy management (IEM). Although multiple value propositions apply to these resources [1], the valorisation of flexibility is developing slowly. Since it is hard to match a value proposition to a flexible resource with specific parameters such as location, size and technology-specific parameters, no off-the-shelf solutions are available.

A review study from 2013 [2] discussed over 200 studies on the evaluation of storage flexibility as a valuable asset. Many attemps have been made to put a value on the flexibility provided by storage technologies and demand response [3][6]. The large number of studies indicates that the valuation of flexibility is complex and can be approached from different points of view.

In contrast to most studies, we take a technology-neutral approach to assess the value of flexibility. We use this approach to develop a framework for quantitive comparison of flexibility valuations for different value propositions and different storage and DR technologies. To enable comparison of technologies and applications, we employ a reference scale of $1 \mathrm{MW}$ installed flexible capacity, and vary other key parameters.

To demonstrate the applicability of the technology-neutral framework, we present a profitability assessment for three value propositions: maximizing self-sufficiency under flat retail prices, ahead market and balancing market participation, where the latter includes reserve markets and imbalance markets. First, the revenue potential for the flexibility in $€ \mathrm{c} / \mathrm{kWh}$ is given for the locations Netherlands, Germany and Italy. This revenue potential can be used as a reference to which results of other studies can be compared. Second, we provide a lower bound estimation of the revenue of selected technologies under more realistic assumptions. The difference between the revenue potential and the realistic lower bound estimation shows the potential value of more intelligent or optimized solutions.

This paper is organised as follows: Section II provides an overview of the literature in valuation of flexibility. The technology-neutral framework is presented in Section III. Section IV presents the assumptions for an optimistic scenario and provides the revenue potential for the retail, ahead and balancing market. The refined evaluation of the least realistically achievable revenue is presented and discussed in Section V. The discussion and conclusion are presented in Section VI.

\section{RELATED WORK}

Multiple value propositions for storage and DR technologies have been evaluated previously: self-sufficiency under feedin tariffs (FIT) [7], ahead market participation [4]-[6], [8], providing ancillary services to system operators [9], [10], flexibility as substitute to conventional grid reinforcements [11] or to curtailment of wind energy [12], [13]. Some papers evaluate the cumulative value of multiple value propositions [14], [15]. Zucker et al. [2] concluded that the results of all these studies are hard to compare due to the large bandwidth of models, assumptions and use cases.

Most studies consider a specific technology such as Li-ion batteries [5] or compressed air energy storage [16]. These works use models that are tailored to a specific technology and value proposition, but less suitable for comparison of different value propositions and technologies. A minority of the works uses a technology-neutral way of assessing the value 
of flexibility. For example, [17] provides a unified framework for assessing different types of DR and storage qualitatively. A quantitative technology-neutral approach is presented by Eyer \& Corey [18] and Miranda et al. [6]. Eyer \& Corey provide a high-level technology-neutral framework for assessing the value of flexibility. The numbers provided in their work are useful for making a first cut when matching technologies and value propositions. In contrast, our work is more specific since we use time series data of existing markets. Miranda et al. provide an upper bound valuation for market participation by energy storage systems in the Iberian, Nordic and Great Britain markets using a Mixed Integer Linear Programming Approach (MILP). In contrast to Miranda et al., we present a technology-neutral model that can represent both storage and demand response technologies. Furthermore, we provide an upper bound that is not constrained by state-of-charge limits and thus applies as a more general insight to any constrained storage.

Most common assumptions in flexibility valuation studies are perfect foresight of prices and that the exploitation of the flexible resource does not have influence on market prices: the price-taker assumption [2]. In this paper, we assume price-taking since we study the value of small-scale storage exploitation. However, we assume perfect foresight of prices for the calculation of the upper bound valuation (an optimistic assumption); for the lower bound valuation (a simple realistic assumption) we explicitly consider a forecasting signal with varying degree of zero-mean normal error.

\section{FRAMEWORK}

This section presents a technology-neutral framework to assess the value of flexibility for a range of flexible resources and value propositions. First, a set of flexibility parameters, representing the most important characteristics of flexible resources in a technology-neutral way is presented. Second, the corresponding optimization problem for intelligent energy management is given for participating in retail, ahead and balancing markets.

\section{A. Flexibility parameters}

A set of technology-neutral flexibility parameters, describing the flexibility provided by a device or an aggregation of devices (such as a microgrid or a virtual power plant) is given in Table I. The flexibility parameters are technology-neutral descriptors, such that many technologies or combinations of technologies could be described. The flexibility parameter set is highly simplified with respect to specific technologies, but provides a generic description of a broad range of technologies that is useful for estimating the value of flexibility.

The flexibility limit $u$ per trading interval $\delta t$, the duration of the energy block that can be bought or sold in a specific market, is mainly determined by the power rating $P$, but also affected by factors as the activation time, ramp rate, local network constraints (such as peak limit and inrush constraints) and response frequency [17]. In our simplified model, we assume $u=\delta t \cdot P$, where $\delta t=\frac{1}{4} h$ for quarter hourly markets and $\delta t=1 h$ for hourly markets, and further $E \geq P \cdot \delta t$.

The energy amount that can be stored in the flexibility resource is mainly determined by the energy rating $E$. For a Demand Response (DR) system, we assume that the energy rating is the maximum shiftable load within a time horizon $T$. Since we pick a reference scale of $1 \mathrm{MW}$ installed flexibility capacity, we neglect market entrance limits that can impede the valorisation of small loads or the fact that sometimes large volumes cannot be matched by the liquidity of the market.

The activation cost function $c_{a}(x)$ returns the one-way cost of using flexibility, capturing e.g. wear-and-tear and losses incurred by charging or discharging a battery. Capturing wearand-tear in this way ensures that we are able to buy a new system after the lifte cycle time has passed by. This implies that we ignore any cash flow requirements. The simulation models presented in this paper assume that the activation cost scales linearly with the activated flexibility volume $x$, i.e. $c_{a}(x)=C_{a} x$, where $C_{a}$ is a constant, thus we neglect nonlinearities due to changing efficiencies or degradation.

To capture the influence of varying degree of error in forecasting, the framework can bear one or more information quality parameters. These parameters describe the correlation between a signal and a stochastic variable that influences the revenue, e.g. the market price or an uncontrollable load the IEM has to balance using the flexible resource. The most influencing stochastic variables for our selected applications (retail, ahead and balancing market participation) are price and load. For the experiments described in Section V, we use zeromean normal errors with standard deviation $\sigma$. The zero-mean assumption is motivated by the fact that systematic biases in prediction can typically be accounted for by mean-adjustment of the predictor. The normal assumption is typical [19], as the specific forecasting method is not subject of this article, and the distribution could easily be replaced in future work.

The revenue of a specific flexibility resource in a given market is represented by $\psi$, the revenue per installed capacity $P$ in $€ / \mathrm{MW} / \mathrm{y}$ and $\varphi$, the revenue per available MWh flexibility in $€ / \mathrm{MWh}$. The revenue $\psi$ can easily be upscaled albeit market liquidity has to be considered outside of our model. Furthermore, the annual shifted volume $Q$ indicates whether the flexibility resource is fully exploited. A low utilization, with $\frac{Q}{\frac{1}{2} V}$ yielding the established utilization factor, suggests looking into combining value propositions.

\section{B. Problem formulation}

We use the technology-neutral parameters formulated in Section III-A to define the optimization problem for intelligent energy management. First, an optimization function is formulated for increasing self-sufficiency under current retail prices. Second, the ahead and balancing value propositions are captured as optimization problems in discrete-time systems. For brevity, we use the notations $[x]^{+} \equiv \max (0, x)$ and $[x]^{-} \equiv \min (0, x)$.

1) Retail market: We consider a scenario where the retail consumption price $p_{c}$ is greater than the feed-in tariff $p_{\text {fit }}$. This 
TABLE I

THE TECHNOLOGY-NEUTRAL PARAMETERS OF FLEXIBILITY FROM STORAGE OR DEMAND RESPONSE OF INDIVIDUAL OR AN AGGREGATION OF DEVICES.

\begin{tabular}{|c|c|c|c|}
\hline Parameter & Symbol & Unit & Description \\
\hline Power rating & $P$ & MW & The highest power input or output allowed to flow through the equipment. \\
\hline Energy rating & $E$ & MWh & The maximum state of charge of a storage system. \\
\hline TI flexibility limit & $u$ & MWh & $\begin{array}{l}\text { Flexibility activation limit in } 1 \text { market time interval TI with duration } \delta t \text {. Typically } u=\delta t \cdot P \text { if } \\
\text { the activation time is smaller than the time to reach the power rating. For example, } u=\frac{1}{4} h \cdot P \\
\text { for quarterly hour markets and } u=1 h \cdot P \text { for hourly markets. }\end{array}$ \\
\hline Flexibility capacity & $V$ & $\mathrm{MWh} / \mathrm{y}$ & Refers to the annual total of rated or reserved flexible capacity, $V=\sum_{t} u_{t}$ \\
\hline Price information quality & $\sigma$ & $€$ & $\begin{array}{l}\text { Assuming zero-mean normal forecast error, standard deviation } \sigma \text { characterizes the information } \\
\text { quality in our model. }\end{array}$ \\
\hline Activated flexibility & $x_{t}$ & MWh & Flexibility activated at $t$ with duration of $d t$. \\
\hline Shifted volume & $Q$ & $\mathrm{MWh} / \mathrm{y}$ & Annual volume of shifted flexibility, $Q \leq \frac{1}{2} \cdot V$. \\
\hline Annual operational profit & $\psi$ & $€ / M W / y$ & $\begin{array}{l}\text { Annual operational profit per installed capacity } P \text {. Intuitively, } \psi \text { indicates how much business } \\
\text { there is per MW per year. }\end{array}$ \\
\hline
\end{tabular}

is in line with the current situation in Amsterdam, Berlin and Palermo. Over the time horizon $T$, the costs of retail market participation are $c_{\mathrm{r}}(\vec{x})$ (Equation 1) and the activation costs are $c_{\mathrm{a}}(\vec{x})=\sum_{t=0}^{T} c_{\mathrm{a}}\left(x_{t}\right)$. In this scenario, the objective for the intelligent energy management system is to minimize Equation 2 , where $\varepsilon_{t}$ is the exogenous supply (respectively demand if negative), and $x_{t}$ is the flexible load activated in trading interval starting at $t$.

$$
\begin{gathered}
c_{\mathrm{r}}(\vec{x})=\sum_{t=0}^{T} p_{c}\left[\varepsilon_{t}-x_{t}\right]^{+}-p_{\mathrm{fit}}\left[\varepsilon_{t}-x_{t}\right]^{+} \\
c_{\mathrm{tot}}(\vec{x})=c_{\mathrm{r}}(\vec{x})+c_{\mathrm{a}}(\vec{x})
\end{gathered}
$$

2) Ahead and balancing markets: For all time steps $t \in T$ the intelligent energy management (IEM) system has to decide what amount of flexibility $x_{t}$ to activate given the flexibility buffer's state of charge $s o c_{t}$, the information coming from a price information signal $h_{t}$ and the exogeneneous load forecast $\hat{\varepsilon}_{t}$. Furthermore, the IEM system has some prior knowlegde such as private cost of flexibility activation $c_{a}\left(x_{t}\right)$ and the overall price distributions. We model this problem as a discrete-time system:

- $S$ contains all possible states of the flexibility resource that are sufficient and necessary to derive the costs and thus constitute the action selection policy. A state $s_{t} \in S$ is described as: $s_{t}=\left(\operatorname{soc}_{t}, \overrightarrow{p_{t}}, h_{t}, \overrightarrow{\varepsilon_{t}}, \hat{\varepsilon}_{t}\right)$, where:

- The set $S O C=[0, E]$ contains all possible state-of charge values. soc $_{t} \in S O C$ is the state of charge just before commencement of the trading interval TI starting at $\mathrm{t}$.

- The prices $\bar{p}_{t}$ and $\underline{p}_{t}$ are the prices for market players that respectively overproduce/ramp up or overconsume/ramp down. In our simulations, these prices follow historic time series but looking at uniform randomly drawn interval $\mathrm{TI}_{t}$, they follow the distributions $P_{u p}$ and $P_{\text {down }}$. The set $\overrightarrow{p_{t}}$ contains all prices $p_{i}$ for $i \leq t$.

- The signal $h_{t}$ is a signal correlated with the real prices. For the simulations presented in this paper, we use $h_{t}=\left(\bar{p}_{t}+\xi_{1}, \underline{p}_{t}+\xi_{2}\right)$, where $\xi_{i} \sim \mathcal{N}(\mu=0, \sigma)$.

- $\mathcal{E}$ contains all possible exogenous loads for $\varepsilon_{t}$. The set $\overrightarrow{\varepsilon_{t}}$ contains all exogeneous loads $\varepsilon_{i}$ for $i \leq t$. For the simulations presented in this paper is assumed $\mathcal{E}=[0]$.

- $\hat{\varepsilon}_{t}$ is the estimation of the exogenous load $\varepsilon_{t}$.

- Actions: $A=X$ is the set of all possible actions $a=x_{t}$, where $x_{t}$ is the flexibility activation in the trading interval starting at $t$.

- Transition: $s_{t+1}=\left(\operatorname{soc}_{t}+x_{t}, \overrightarrow{p_{t+1}}, h_{t+1}, \overrightarrow{\varepsilon_{t+1}}, \hat{\varepsilon}_{t+1}\right)$ describes the transition from $s_{t}$ to $s_{t+1}$ given action $a_{t}$.

- Objective is to minimize the cost function:

$$
\begin{array}{r}
\min _{\vec{x}}\left(\sum_{t=0}^{T} c_{t}\left(x_{t}\right)+\sum_{t=0}^{T} \underline{p}_{t}\left[\epsilon_{t}-x_{t}\right]^{-}-\sum_{t=0}^{T} \bar{p}_{t}\left[\epsilon_{t}-x_{t}\right]^{+}\right) \\
\text {subject to } \sum_{t=0}^{T} x_{t}=0
\end{array}
$$

\section{ReVEnUe POTENTIAL}

In order to determine the revenue potential in a market, i.e. the upper bound revenue, we calculate revenue under very optimistic assumptions. First, all loads can be shifted freely throughout the time horizon $T$ under consideration. In this case the energy rating is not limiting the use of flexibility, i.e. $E \geq P \cdot T$. Second, we assume no activation cost and third, $100 \%$ round trip efficiency. 


\section{A. Retail}

In this section, we consider a typical tariff from the endcustomer retail market. Consumption is charged $p_{c}$ and feed-in $p_{\text {FIT }}$. The tariff considered here does not apply annual netting, which is in line with the current situation in Germany and Italy, but in constrast to the situation for fuses $<3 \times 80 A$ in the Netherlands, which still has this regulation in place that removes any IEM incentives.

Since we assume no limit on storage capacity, the optimal policy for retail market participation is to shift local demand that is not matched with local supply and vice versa. For every new match the gain is the difference between the retail price $p_{c}$ and the feed-in-tariff $p_{\text {FIT }}$. Table II shows our estimated for this value for the Dutch, German and Italian retail market. The retail price data as provided by CBS (Statistics Netherlands) [20] is used for the Netherlands. The ratio between the household tariffs in Germany [21] and Italy [22] ${ }^{1}$ and the Netherlands is used to estimate the prices in Germany and Italy for the larger scales. As feed-in tariffs we take the annual average solar feed-in price (Netherlands [23], Germany [24] and Italy [25]). In this particular tariff, the marginal profit per kWh only depends on the price difference, and the annual profit on the shiftable volume, which is bound by the volume of unmatched local demand and supply. In turn, this mismatch is bound by total demand, which is used in Table III to compute annual potential value. In this table we used an estimate of the demand volume in a typical apartment building (60 dwellings), office environment and small industry with on-site production.

\section{B. Ahead or balancing market participation}

In this section, we consider ahead, imbalance and reserve market participation. Although market designs are different, the decision problem for flexible resources participating in these markets is similar and can be described by the problem formulation presented in Section III-B2. We study the revenue potential of these markets under the assumptions that the IEM operator is a price-taker and that it has perfect forecast of all market prices in $T$.

Given a historic time series of market prices for buying $\underline{p}_{t}$ and selling $\bar{p}_{t}$, the optimal policy in the optimistic scenario for market participation is to buy all residual shiftable loads from the market for the $n$ lowest prices and sell them again to the market for the $n$ highest prices, where the $n$th trade is the last trade that generates an operational profit, such that when matching intervals $i$ and $j, \bar{p}_{i}-\underline{p}_{j}>0$. As a consequence of the price-taker and perfect forecast assumption, the optimal solution is $\forall t, x_{t} \in\{-u, 0, u\}$. This equilibrium solution neglects that each interval can only be activated in one direction, however it provides an very efficient computation for an upper bound, and given correlations between $\underline{p}_{t}$ and $\bar{p}_{t}$ the equilibrium is likely to contain few if any double bookings. In our simulation, we observed few such occurences $(<0.01 \%)$

\footnotetext{
${ }^{1}$ In Italy day-night tariffs for households are more common, therefore the constant tariffs are relatively high compared to the Amsterdam and Berlin region.
}

TABLE II

THE VALUE OF FLEXIBILITY IN EURO CENTS PER SHIFTED KWH AS DESCRIBED IN SECTIONS IV-A AND IV-B.

\begin{tabular}{llrrr}
\hline Market & Segment & Netherlands & Germany & Italy \\
\hline Retail & Apartment & 9.0 & 12.0 & 10.0 \\
& Office & 1.0 & 2.0 & 2.0 \\
& Industry & 0.2 & 1.0 & 0.3 \\
\hline Ahead & Day-ahead & 2.1 & 2.5 & $5.6^{* *}$ \\
Balancing & Imbalance & 7.7 & 9.4 & - \\
& Reserve & 10.4 & $*$ & - \\
\hline
\end{tabular}

*Incentives are available but this case is omitted due to weekly commitment requirement. $* *$ Sicily, Italy.

TABLE III

COMPARISON OF UPPER BOUNDS FOR POTENTIAL ANNUAL VALUE OF ZERO-COST FLEXIBILITY IN K€ WITH SHIFTABLE VOLUME Q IN GWH.

\begin{tabular}{llrrrrrr}
\hline \multirow{2}{*}{ Market } & \multicolumn{2}{c}{ Netherlands } & \multicolumn{2}{c}{ Germany } & \multicolumn{3}{c}{ Italy } \\
& Segment & $\mathrm{Q}$ & $\psi$ & $\mathrm{Q}$ & $\psi$ & $\mathrm{Q}$ & $\psi$ \\
\hline \multirow{2}{*}{ Retail } & Apartment & 0.1 & 1.2 & 0.1 & 1.7 & 0.1 & 1.4 \\
& Office & 0.4 & 0.4 & 0.4 & 0.9 & 0.4 & 0.9 \\
& Industry & 5.3 & 1.0 & 5.3 & 5.3 & 5.3 & 1.6 \\
\hline \multirow{2}{*}{ Ahead } & Day-ahead & 4.4 & 91.7 & 4.4 & 107.4 & 6.0 & $337.9 * *$ \\
Balancing & Imbalance & 3.9 & 302.2 & 4.4 & 412.5 & - & - \\
& Reserve & 5.5 & 577.2 & $*$ & $*$ & - & - \\
\hline
\end{tabular}

for the Dutch and German day ahead and imbalance markets if $\sigma=0$, and if $\sigma$ is large, double bookings only increase slightly. For the Dutch reserve market and the Italian day ahead market, the double booking percentage is $36 \%$ and $28 \%$ respectively, and thus provide more optimistic overestimating bounds. The refined solution in the next section is by design immune to such infeasible allocations.

The optimal solution can be found by pairing ramp up and ramp down prices $\forall t, \bar{p}_{t}$ and $\forall t, \underline{p}_{t}$ greedily. When we consider each time interval $t$ as an individual trader that submits $\bar{p}_{t}$ as a bid and $\underline{p}_{t}$ as an ask to a double auction, the solution is equal to the Nash equilibrium. ${ }^{2}$ Results are juxtaposed to the retail values in Tables II and III.

\section{REFinED EVALUATION}

After estimating the upper revenue limits in the previous section, we now use the flexibility parameters to give a more refined estimation of the revenue that can be achieved by intelligent energy management. First, we use our parameterization to model the flexibility of a specific technology (the Durion CS 55/123 battery), and provide a realistic lower bound revenue estimation for this technology in a specific target market (passive market participation in the Dutch imbalance market). Second, we explore how the parameters of the flexible resource more generally influence its revenue in that market.

\section{A. Case study: Durion battery}

Table IV shows the economical and technical properties of a package of four Durion CS 55/123 batteries (LiFePO4)

\footnotetext{
${ }^{2}$ Proof omitted but trivial by contradiction. Intuitively, if an interval is selected for flexibility activation, any activation below the limit could be improved in profit by going to the limit, considering a few boundary cases.
} 
[26] linked in parallel, and the flexibility parameters for this package that were derived from these properties.

We evaluate a simple heuristic policy, since this provides a realistic lower bound of the achievable revenue. The heuristic policy (Equation 3) evaluates what the estimated operational profit of a one-way activation of flexibility is. If the estimated profit exceeds the cost of activating the flexibility, the flexibility is activated.

$$
\text { buy if } \begin{aligned}
\underline{h}_{t}+2 C & \geq \mathrm{E}\left(\bar{p}_{t}\right) \\
\text { sell if } \bar{h}_{t} & \leq \mathrm{E}\left(\underline{p}_{t}\right)
\end{aligned}
$$

This heuristic is a natural match for imbalance markets, where prices are defined after the fact. Only the signal $\left(\bar{h}_{t}, \underline{h}_{t}\right)$ is available, which provides the estimated value of $\left(\bar{p}_{t}, \underline{p}_{t}\right)$. It also captures ahead and reserve markets, since volumes have to be precommitted before market clearing.

Figure 1 shows the estimated revenue for a set of Durion battery packages, with a total of $1 \mathrm{MW}$ installed flexibility capacity. The investment cost per $1 \mathrm{MW}$ installed flexibility is $18.2 \cdot 476 \mathrm{k}=8.7 \mathrm{M}$ and the upper bound revenue of market participation is $90 k € / y$. This results in a break-even time of 96 year even while neglecting the P/E ratio and other constraints. In this case the battery was replaced every 2-3 years, so it would not expire. Figure 1 shows that if the energy rating is neglected $(E \geq P \cdot T)$ the performance of the heuristic is close to the performance of the offline algorithm and even better when the forecasting error is large. This indicates that there is little gain from intelligent energy management for situations with high activation costs and small $\mathrm{P} / \mathrm{E}$ ratio beyond the achievable performance of a simple heurstic. The breakeven point for the specific Durion battery package, limited by $\mathrm{P} / \mathrm{E}$ ratio $=0.15$, is after 176 years. This indicates that a higher $\mathrm{P} / \mathrm{E}$ ratio results in lower cost-effectiveness.

\section{B. Influence of flexibility parameters}

The previous section has shown that the investment cost and thus the activation costs of an example storage technology that is currently on the market is too high to generate profit from

TABLE IV

ECONOMICAL AND TECHNICAL PARAMETERS OF THE A PACKAGE OF FOUR DURION CS 55/123 BATTERIES [26].

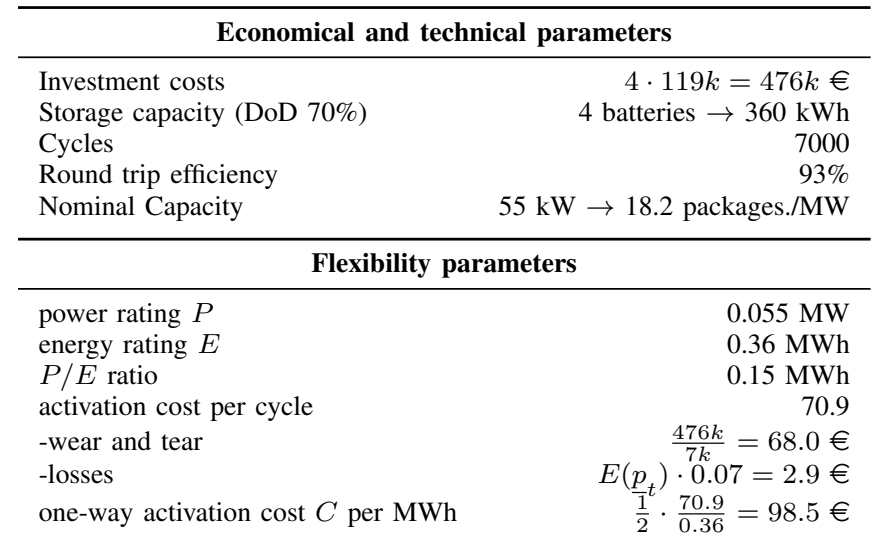

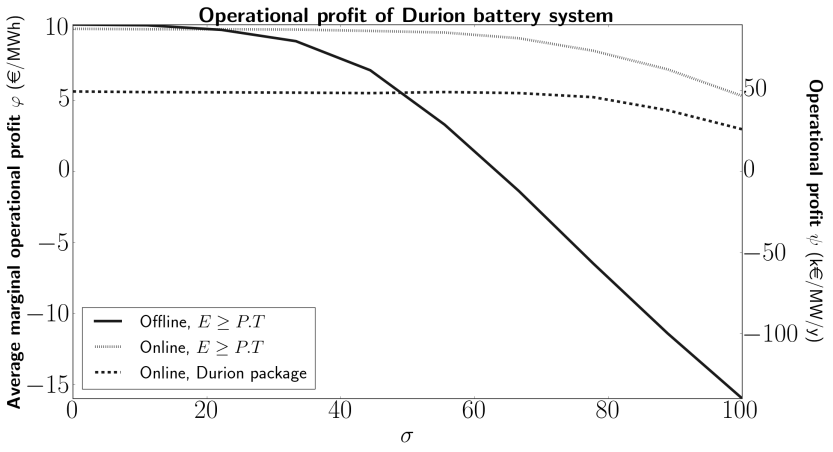

Fig. 1. Revenue estimation for the heuristic online policy for imbalance market participation using the flexibility of the Durion CS 55/123 battery package. The characteristic of this flexible resource are in Table IV. The revenue is estimated by averaging over 10 simulations with randomized forecasts.

intelligent participation in the Dutch imbalance market. In this section we explore the characteristics of flexible resources more broadly.

Figure 2 shows how the activation costs and the quality of the forecasting signal influence the performance of a flexibility buffer with the characteristic: $E \geq P \cdot T$. Figure 2 can be used to find what the maximal investment and/or operational costs of a flexible resource can be. For example, if the activation cost is $40 € / \mathrm{MWh}$, the revenue for $\sigma<30$ yields $140 \mathrm{k} € / \mathrm{y}$. For a resource with a life time of 10 years, the maximum investment cost is $1.4 \mathrm{M} € / \mathrm{MW}$.

An interesting observation is that a signal quality $\sigma<40$ is sufficient: better signal quality does not influence the revenue much. The highest operational profit is found for zero activation cost and $\sigma=30$, which indicates that the heuristic policy is not optimal and that there is a niche for developing more intelligent policies. A typical price signal for the prices at $t$ could be the price at $t-1$. The price difference between these time slots is not well approximated by a normal distribution, therefore it is not advisible to compare this price signal to the signals used in our simulation using $\sigma$. However, we can use Spearman's $r$, a rank-based correlation coefficient for comparison. The typical $t-1$ prediction signal yields a comparable $r$ as a normal signal with $\sigma$ in the range of $50-60$.

\section{DISCUSSION \& CONCLUSION}

In this work we give guidance on the valuation of flexibility as provided by Demand Response or storage in electricity markets. We propose a technology-neutral description of flexibility, that enables us to compare the revenue potential of different resources providing flexibility. We use this technology-neutral description to provide a $€ \mathrm{c} / \mathrm{kWh}$ and a $€ / M W / y$ valuation of flexibility. First, an upper bound for the value of flexibility in the retail, ahead and balancing markets in the Netherlands, Germany and Italy has been computed under optimistic assumptions, providing a guide for attention for business opportunities, and a computational method for further exploration. Second, an evaluation of a 


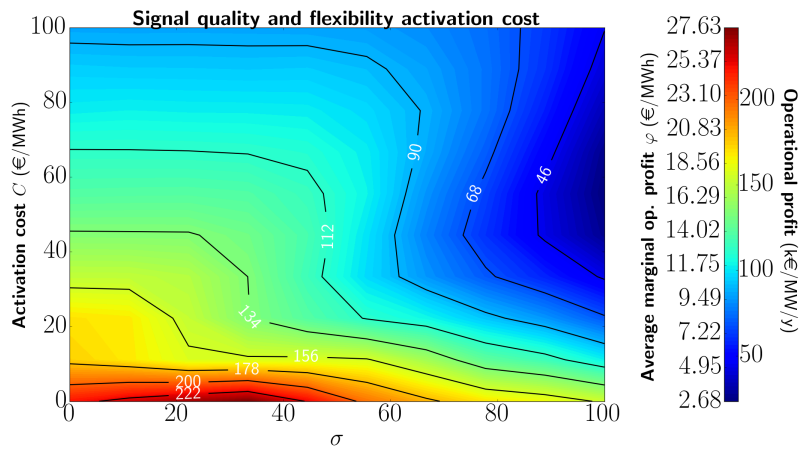

Fig. 2. Revenue estimation for the simple heuristic online policy for participation in the Dutch imbalance market for varying activation costs and forecasting error. Since $E \geq P . T$, the result is not constrained by the $\mathrm{P} / \mathrm{E}$ ratio.

simple heurstic online policy provides a realistic lower bound for the achievable profits from flexibility over various marginal costs of activation. Finally, the technology-neutral approach is demonstrated on the specific technology of a battery that is currently available on the market.

Our results can serve as a reference simulation and comparison method to more refined and more specific technoeconomical assesments of flexibility resources. Furthermore, the results can be compared to the revenue potential of other value propositions for valorising flexibility, such as flexibility as substitute to curtailment of wind energy [12], [13] or conventional grid reinforcements [11]. Finally, marketparticipation policies for intelligent energy management can be evaluated against the upper bound and realistic lower bound as a benchmark.

Our results suggest that there are business opportunities for flexible resources with low activation costs, which may be achieved by innovative tariffs for demand response. Such innovative tariffs may eventually provide the lacking incentives for the untapped distributed flexibility to contribute to the efficient balancing of our future energy system.

\section{REFERENCES}

[1] M. Zidar, P. S. Georgilakis, N. D. Hatziargyriou, T. Capuder, and D. Skrlec, "Review of energy storage allocation in power distribution networks: applications, methods and future research," IET Generation, Transmission \& Distribution, vol. 10, no. 3, 2016.

[2] A. Zucker, A. Hinchliffe, and A. Spisto, Assessing Storage Value in Electricity Markets: A Literature Review. Publications Office of the European Union, 2013.

[3] G. Jötten, A. Weidlich, L. Filipova-Neumann, and A. Schuller, "Assessment of flexible demand response business cases in the smart grid," in Proceedings of the 21st International Conference on Electricity Distribution, Frankfurt, 2011.

[4] V. François-Lavet, R. Fonteneau, and D. Ernst, "Using approximate dynamic programming for estimating the revenues of a hydrogen-based high-capacity storage device," in IEEE Symposium on Adaptive Dynamic Programming and Reinforcement Learning (ADPRL), 2014.

[5] F. J. Heredia, J. Riera, M. Mata, J. Escuer, and J. Romeu, "Economic analysis of battery electric storage systems operating in electricity markets," in 12th International Conference on the European Energy Market (EEM), 2015.
[6] I. Miranda, N. Silva, and A. M. Bernardo, "Assessment of the potential of battery energy storage systems in current european markets designs," in 12th International Conference on the European Energy Market (EEM), 2015.

[7] J. Weniger, J. Bergner, T. Tjaden, and V. Quaschning, "Economics of residential PV battery systems in the self-consumption age," in 29th European Photovoltaic Solar Energy Conference and Exhibition, Amsterdam, 2014.

[8] N. Löhndorf and S. Minner, "Optimal day-ahead trading and storage of renewable energies: an approximate dynamic programming approach," Energy Systems, vol. 1, no. 1, pp. 61-77, 2010.

[9] R. J. Bessa, M. A. Matos, F. J. Soares, and J. A. P. Lopes, "Optimized bidding of a EV aggregation agent in the electricity market," IEEE Transactions on Smart Grid, vol. 3, no. 1, pp. 443-452, 2012.

[10] B. Biegel, L. H. Hansen, J. Stoustrup, P. Andersen, and S. Harbo, "Value of flexible consumption in the electricity markets," Energy, vol. 66, pp. $354-362,2014$.

[11] S. Nykamp, V. Bakker, A. Molderink, J. L. Hurink, and G. J. M. Smit, "Break-even analysis for the storage of PV in power distribution grids," International Journal of Energy Research, vol. 38, no. 9, pp. 1112-1128, 2014.

[12] J. X. Johnson, R. De Kleine, and G. A. Keoleian, "Assessment of energy storage for transmission-constrained wind," Applied Energy, vol. 124, pp. 377-388, 2014.

[13] E. McKenna, P. Grunewald, and M. Thomson, "Going with the wind: temporal characteristics of potential wind curtailment in Ireland in 2020 and opportunities for demand response," IET Renewable Power Generation, vol. 9, no. 1, pp. 66-77, 2015.

[14] P. Denholm and R. Sioshansi, "The value of compressed air energy storage with wind in transmission-constrained electric power systems," Energy Policy, vol. 37, no. 8, pp. 3149-3158, 2009.

[15] E. Drury, P. Denholm, and R. Sioshansi, "The value of compressed air energy storage in energy and reserve markets," Energy, vol. 36, no. 8, pp. 4959-4973, 2011.

[16] H. Lund, G. Salgi, B. Elmegaard, and A. N. Andersen, "Optimal operation strategies of compressed air energy storage (CAES) on electricity spot markets with fluctuating prices," Applied thermal engineering, vol. 29, no. 5, pp. 799-806, 2009.

[17] F. Oldewurtel, T. Borsche, M. Bucher, P. Fortenbacher, M. Gonzalez Vaya, T. Haring, J. L. Mathieu, O. Mégel, E. Vrettos, and G. Andersson, "A framework for and assessment of demand response and energy storage in power systems," in IREP Symposium on Bulk Power System Dynamics and Control-IX Optimization, Security and Control of the Emerging Power Grid, 2013.

[18] J. Eyer and G. Corey, "Energy storage for the electricity grid: Benefits and market potential assessment guide," Sandia National Laboratories, Tech. Rep. SAND2010-0815, 2010.

[19] J. Contreras, R. Espinola, F. J. Nogales, and A. J. Conejo, "Arima models to predict next-day electricity prices," IEEE Transactions on Power Systems, vol. 18, no. 3, pp. 1014-1020, 2003.

[20] CBS. Elektricity, mean prices for consumers. [Online]. Available: http: //statline.cbs.nl/Statweb/publication/?DM=SLNL\&PA=81309NED\& $\mathrm{D} 1=8-12 \& \mathrm{D} 2=\mathrm{a} \& \mathrm{D} 3=\mathrm{a} \& \mathrm{D} 4=1 \& \mathrm{HDR}=\mathrm{T} \& \mathrm{STB}=\mathrm{G} 2, \mathrm{G} 3, \mathrm{G} 1 \& \mathrm{VW}=\mathrm{T}$

[21] Vattenfall. Electricity consumption tariffs. [Online]. Available: https: //www.vattenfall.de/de/strom-berlin.htm

[22] Segugio. Comparing electricity consumption tariffs. [Online]. Available: http://tariffe.segugio.it/ costo-energia-elettrica/lista-offerte-energia-elettrica.aspx? ricercaEnergia=953da934-9372-4142-9c79-3cd4ba0b9690\& prodottoEnergia $=$

[23] Rijksdienst voor Ondernemend Nederland. (2014) Sde+ 2014: Instructions on how to apply for a subsidy for the production of renewable energy. [Online]. Available: http://www.rvo.nl/sites/ default/files/2014/09/DigitalebrochureSDE+20oktober2014kleur.pdf

[24] Bundesnetzagentur. Germany: feed-in tariffs for PV. [Online]. Available: http://www.bundesnetzagentur.de/SharedDocs/Downloads/DE/ Sachgebiete/Energie/Unternehmen_Institutionen/ErneuerbareEnergien/ Photovoltaik/Datenmeldungen/EEG_Saetze_Apr

[25] PV Magazine. Feed-in tariffs for various countries. [Online]. Available: http://www.pv-magazine.com/services/feed-in-tariffs/ feed-in-tariffs-for-various-countries

[26] PV Magazine. Market overview storage systems. [Online]. Available: http://www.pv-magazine.com/fileadmin/PDFs/Storage_Database.pdf 